over 48 hours increased the epithelial cell proliferation rate by up to $56 \%$ in Caco- $2(\mathrm{p}<0.01)$ and $42 \%$ in HT-29 $(\mathrm{p}<0.001)$ cells.

Conclusion Our data demonstrates that IL-27 enhances epithelial barrier wound healing. Gene expression data suggests that cell-cell adhesion is enhanced through increased E-cadherin expression, with a reduction in permeability through decreased expression of claudin-2 (pore forming) and increase in claudin-4 (pore closing). Tight junction function is enhanced through increased expression of occludin and tight junctional protein-1. Further studies will define the IL-27 driven permeability related protein expression profile and impact on functional permeability in organoids and whether IL-27 is a potential new treatment for IBD.

\section{PWE-011 THE PSYCHOSOCIAL EFFECTS OF INFLAMMATORY BOWEL DISEASE ON REPRODUCTIVE HEALTH - A SYSTEMATIC LITERATURE REVIEW}

\footnotetext{
${ }^{1}$ Satvinder Purewal ${ }^{*}$, ${ }^{2}$ Wladyslawa Czuber-Dochan, ${ }^{3}$ Sarah Chapman, ${ }^{4}$ Christian Selinger, ${ }^{5}$ Helen Steed, ${ }^{5}$ Matthew Brookes*. 'University of Wolverhampton, Wolverhampton, UK; ${ }^{2}$ King's College London, London, UK; ${ }^{3}$ University of Bath, Bath, UK; ${ }^{4}$ Leeds Teaching Hospitals Trust, Leeds, UK; ${ }^{5}$ Royal Wolverhampton Nhs Trust, Wolverhampton, UK
}

\subsection{6/gutjnl-2018-BSGAbstracts. 143}

Introduction Inflammatory bowel disease (IBD) is a chronic condition that can affect patients during their reproductive years. Previous studies report that IBD patients have high levels of pregnancy-related fears and voluntary childlessness. The aim of this project is to perform a literature review on the psychosocial effects of IBD on patient's reproductive health and investigate factors affecting family planning decisions.

Method Six electronic databases (CINAHL, PsycInfo, EMBASE, Pubmed, Web of Science, Sciencedirect) were searched using a broad search strategy. Studies using qualitative, quantitative and mixed methods designs were eligible.

Results Using Prisma-P, a total of 3600 records were identified through electronic databases, hand searching and contacting authors. After removing duplicates, 1806 titles were screened and 241 abstracts were reviewed. Of these, 79 full text articles were screened and 41 articles have been included. The studies design included cross-sectional surveys, qualitative, mixed methods and non-randomised controlled intervention studies. Synthesis of the data revealed that sexual dysfunction after surgery, specifically ileal pouch-anal anastomosis, is common for female patients. A small minority of female IBD patients do not use contraception and are at 'risk' for pregnancy. Knowledge regarding the effects of IBD on pregnancy and fertility is consistently poor and poor knowledge is associated with voluntary childlessness. Many patients report pregnancy-related fears and anxieties including concerns that IBD or medications may harm the baby or lead to a complicated pregnancy. Patients feared transmission of IBD to their offspring, which may lead to voluntary childlessness. Strategies to improve pregnancy and fertility knowledge (e.g., pre-conception counselling) are successful in reducing pregnancy-related anxieties. However, provisions of pre-conception counselling tend to be limited. The literature in this field is associated with a number of limitations including (a) small sample sizes; (b) low response rate for surveys; (c) the use of unstandardised and non-validated questionnaires; (d) few studies have included male samples and ethnic minority groups; and (e) lack of qualitative enquiry and longitudinal follow-up of patients.

Conclusion The literature indicates that some patients with IBD experience sexual dysfunction, poor fertility and pregnancy-related knowledge, high levels of pregnancy-related fears, concerns and voluntary childlessness. Intervention to improve knowledge tends to be successful. This review has identified several psychosocial effects of IBD on reproductive health which need further investigation.

\section{PWE-012 SMALL BOWEL ULTRASOUND IN CROHN'S DISEASE- OUTCOMES IN A DISTRICT GENERAL HOSPITAL}

Robert Perry*, Chia Sin Chey, Hein Htet, Fern Chilcott, Beverley Kirkham, Sarah Langlands, Gauraang Bhatnagar, Jian Wu. Frimley Health NHS Foundation Trust, Camberley, UK

\subsection{6/gutjnl-2018-BSGAbstracts. 144}

Introduction The joint ECCO and ESGAR evidence-based consensus guidelines for imaging techniques for inflammatory bowel disease IBD assessment recommends ultrasound (US) as one of the first-line tests for the investigation of Crohn's Disease (CD). It is inexpensive, free of ionising-radiation and well tolerated. We looked at outcomes in SB US in our CD population.

Methods Retrospective analysis of SB US for patients with known or suspected CD between June 2016 to February 2017 in Frimley Park Hospital. Data was collected from PACS, clinic letters and endoscopy reports.

Result 91 US scans in a total of 83 patients were performed by a single, dedicated GI radiologist (6 patients had more than one US). Patient age range 7-80 years (median 29 years); 53 female (64\%), 30 male (36\%).

21/91 (23\%) US were performed for assessment of symptomatic flare in those with established CD. 16/21 (76\%) had active disease on US (81\% terminal ileitis; $6.3 \%$ stricture, $6.3 \%$ fistula, $6.3 \%$ abscess). Of these, 4 had MRE and 2 had colonoscopy which correlated with US findings. 11/16 (69\%) had treatment escalation following US (55\% started anti-TNF, $18 \%$ steroids, 9\% Vedolizumab, 9\% enteral, 9\% surgery). US was the sole investigation prior to treatment escalation in 7 these patients $(64 \%)$.

24/91 (26\%) US were performed in established CD patients to aid treatment decisions; 4 after recent steroid course (all started disease modifying treatment), 8 to assess patients on biologics, 2 to evaluate starting biologics, 6 to evaluate previous abnormal/inconclusive CT/MRI or colonoscopy, 2 periprocedurally, 1 for discordant symptoms and imaging; 1 for abnormal biochemistry.

6 US were undertaken after failure of terminal ileum intubation for established CD. 4/6 (67\%) detected terminal ileitis and treatment subsequently escalated (1 started methotrexate, 1 anti-TNF, 1 Vedolizumab, 1 prednisolone).

46/91 (51\%) US were performed for suspected CD. 11/46 (24\%) showed active inflammation. 8 were ultimately diagnosed with CD. In this group, 2 had MRE, 3 had colonoscopy and 3 had both, all correlating with US findings. 35/46 (76\%) did not show active inflammation but reported incidental findings including malignancy and gallstones.

Conclusion This study demonstrates the useful role of SB US in the management of CD. Our results show that US led to changes in treatment including management of acute flares, 